\title{
Robotic Monitoring of Power Systems
}

\author{
Bing Jiang, Student Member, IEEE, and Alexander Mamishev, Member, IEEE
}

\begin{abstract}
Monitoring of electric power systems in real time for reliability, aging status, and presence of incipient faults requires distributed and centralized processing of large amounts of data from distributed sensor networks. To solve this task, cohesive multidisciplinary efforts are needed from such fields as sensing, signal processing, control, communications, optimization theory, and, more recently, robotics. This review paper focuses on one trend of power system monitoring, namely, mobile monitoring. The developments in robotic maintenance for power systems indicate significant potential of this technological approach. Authors discuss integration of several important relevant sensor technologies that are used to monitor power systems, including acoustic sensing, fringing electric field sensing, and infrared sensing.
\end{abstract}

Index Terms-Automated maintenance, distribution power systems, mobile sensing, real-time monitoring, sensor arrays.

\section{INTRODUCTION}

$\mathbf{E}$ CONOMICALLY effective maintenance and monitoring of power systems to ensure high quality and reliability of electric power supplied to customers is becoming one of the most significant tasks of today's power industry. As with any preventive maintenance technology, the efforts spent on the status monitoring are justified by the reduction of the fault occurrence and elimination of consequent losses due to disruption of electric power, damage to equipment, and emergency equipment replacement costs.

In the past few years, there have been several significant developments on monitoring technologies for distribution power cables. This review describes technical results relevant to mobile sensing of distributed systems, especially for maintenance tasks.

\section{BACKGROUND}

It is a well-recognized fact in surveillance and monitoring fields that measurement of parameters of a distributed system has inherently higher resolution and accuracy when it is accomplished with a scanning device as opposed to a wide-angle global system. Experimentally, this has been confirmed in numerous cable testing studies (e.g., [1]). This principle remains in force for almost all sensing techniques: it is possible to monitor a distributed system with a global device; for example, using terminal characteristics or propagating waves, but this approach

Manuscript received November 28, 2001. This work was supported in part by National Science Foundation CAREER Award 0093716 and in part by the Electrical Energy Industrial Consortium and Advanced Power Technologies (APT) Center at the University of Washington. The APT Center is supported by RTE, AREVA, PJM, CESI, LG Industrial Systems, and Mitsubishi Electric Corp.

The authors are with the Sensors, Energy, and Automation Laboratory (SEAL), Department of Electrical Engineering, University of Washington, Seattle, WA 98195 USA (e-mail: mamishev@ee.washington.edu).

Digital Object Identifier 10.1109/TPWRD.2004.829918 does not match the resolution of equivalent sensors placed in the direct vicinity of system components.

In addition to sensitivity improvement and subsequent system reliability enhancement, the use of robotic platforms for power system maintenance has many other advantages. Replacing human workers for dangerous and highly specialized operations, such as live maintenance of high-voltage transmission lines, has been a long-standing effort in the power community. Other needs of robotics in power systems include operation in hazardous environments, such as radioactive locations in nuclear plants, access to tight spaces, such as cable viaducts and cooling pipes, and precise positioning of measurement equipment. Therefore, one may expect that the mobile sensing will play an increasingly important role in the monitoring of power systems.

Numerous worldwide projects attacked this challenging application from different angles. In 1989, two manipulator systems differing in operating method were developed by Tokyo Electric Power Company to traverse and monitor fiber-optic overhead ground transmission wires (OPGW) above 66-kV power transmission lines [2]. It was shown that the systems are fully capable of performing distribution line construction work using stereoscopic TV camera system. Several other teleoperated robots have been developed for live-line maintenance in Japan [3], [4], Canada [5], Spain [6], and other locations.

An autonomous mobile robot was developed to inspect the power transmission lines in 1991 [7]. The robot can maneuver over obstructions created by subsidiary equipment on the ground wire. It is equipped with an arc-shaped arm that acts as a guide rail and allows it to negotiate transmission towers. At the same time, a basic synthesis concept of an inspection robot was described for electric power cables of railways [8]. Since the feeder cables are extremely long and have many irregular points, a multicar structure with joint connections and biological control architecture was adopted; thus, the robot could run on the cable smoothly with sufficient speed and deal with the shape irregularities.

\section{TECHNOLOGICAL NEEDS}

Hardly any successful robot applications have been reported for underground distribution cables. Numerous problems have to be solved for this kind of a robot, such as space confinement, size and weight restrictions, wireless design requirements, and adverse environmental conditions. Miniaturization has been one of the most difficult problems. With the continuing development of MEMS, microelectronics, and communication technologies, this problem is on the verge of being solved. Successful applications of microbots were demonstrated in other fields (e.g., [9]). Fig. 1 shows a conceptual design of a mobile platform, a modular system with a separate unit providing autonomy of opera- 


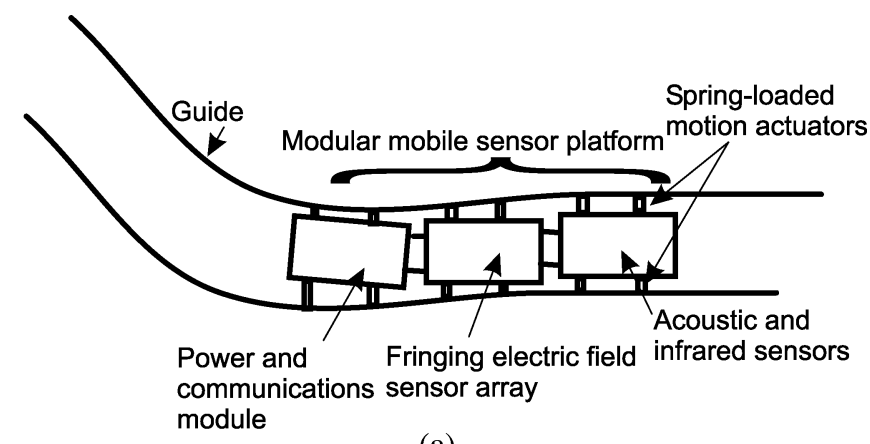

(a)

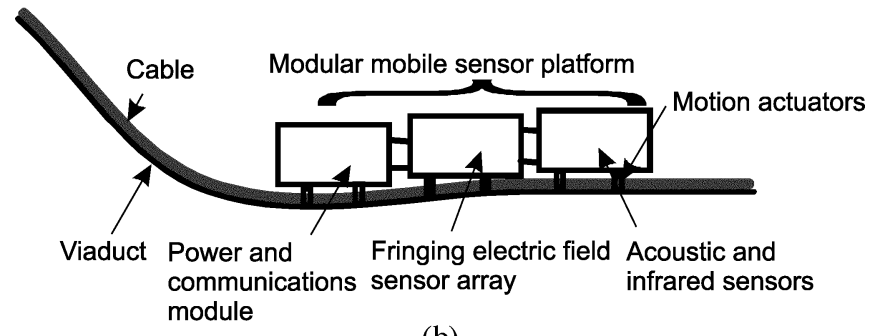

(b)

Fig. 1. Miniature robotic platform for monitoring of transmission and distribution power cables. (a) Internal platform. (b) External platform.

tion and reconfigurable sensing arrays in a master/slave arrangement.

Generally speaking, the mobile monitoring of power systems involves the following issues.

1) Sensor fusion: Monitoring the condition of cables requires incorporation of sophisticated property-monitoring sensors in addition to positioning, tactile, and other sensors aimed to support the autonomy of robot movement.

2) Motion pattern: Inspection robots used in power systems can be subdivided into external and internal ones (see Fig. 1). External robots travel over the outer surface of electrical components and may possess a high degree of autonomy [8], whereas internal units use inner spaces of ducts and pipes and are often implemented as track-following devices with a predetermined route, and a limited set of operations [10]. The level of autonomy depends on the task. For example, routine inspection and maintenance require a high degree of autonomy for economical reasons.

3) Power supply: Since the cable network is a global distributed system, it is very limiting for the inspecting robot to draw a power cord behind itself. Ideally, the power supply has to be wireless. It is desirable that the platform harvest energy from energized cables. Inductive coupling for a wireless power supply could be a desired method. It has been investigated for vehicles [11], batteries [12], microsystems [13], and numerous consumer applications. Although a low-frequency coupling is less efficient than a microwave mode [14], direct proximity to the power cable will make it a viable choice. Of course, the platform requires an independent backup power source as well.

4) Control strategy: It includes object tracking, collision avoidance, and prevention of electrical short circuits. The control system receives initiating commands from the operator for the global tasks, and small tasks are often preprogrammed. The most important requirements are the following.

a) The control should be robust because of complicated motion requirement and the irregular surface of the cable connections.

b) It should include an optimum algorithm used to locate the sensor array with respect to the inspected system, a path planning algorithm used to track the whole or part of the network with the shortest path, and control sequences adaptively switching sensor operation from a fast superficial inspection mode to a slow detailed inspection mode.

c) The robot requires considerable computational resources to be adaptive and flexible. This fact is highly problematic because of the limited size of the robot, especially for underground applications. Accordingly, this strongly argues for the use of communication and offboard intelligence. This also involves allocation between local and remote signal processing.

5) Communication: The communication module exchanges data between the master computer and the mobile robot, including data originating from different streams on both sides of the communication link and different priorities associated with it. A multiplexing problem that concerns the allocation between local and remote computation capacity has to be solved too [15].

6) Positioning system: It should work like the Global Positioning System (GPS), used to estimate the location of the robot. Therefore, effective maintenance and rescue tasks for cable systems, even for the robot itself, can be carried out. In most applications, two basic position estimations are employed: relative and absolute positioning. Relative positioning can provide rough location estimate, the absolute one can compensate the errors introduced [16].

\section{SENSORS AND Signal PROCESSING}

The main sensing principles appropriate for monitoring of power cables include acoustics, dielectrometry, thermal imaging, eddy currents, and visual inspection. This section offers an overview of several projects dealing with relevant technological issues.

\section{A. Discrimination of Energized Cables}

As the power network continues to expand and becomes more interconnected, the task of maintaining an accurate topological representation of a power grid is often sacrificed. Consequently, maintenance personnel often need to determine the energization status of underground cables. A mobile monitoring system should be capable to do the same task.

The predominant detection method practiced today is intrusive in nature, requiring the maintenance personnel either to puncture or remove the protective conducting sheath surrounding the cable in order to measure the emanating electric field [17]. This method is dangerous since the drill bit may reach the conductors unexpectedly. More significantly, 


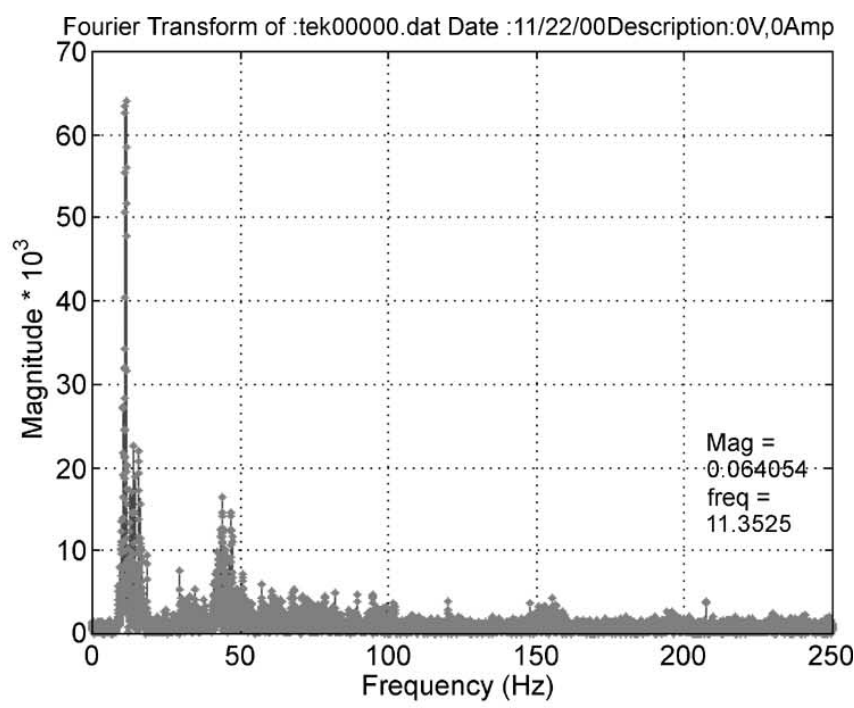

(a)

Fourier Transform of :tek00000.dat Date :11/22/00Description:143.3.3V,10.56Amp

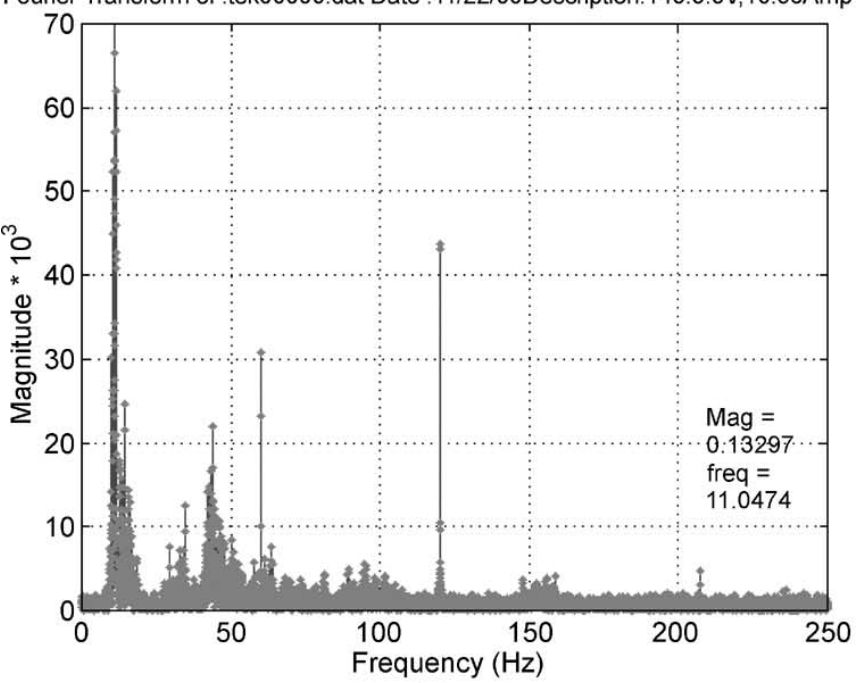

(b)

Fig. 2. Fourier transform of acoustic signatures of underground power cables. (a) Nonenergized cable. (b) Energized and loaded cable.

this method compromises the integrity of cable insulation. Acoustic vibrations generated by coulombic forces and current interactions are a preferred potential indicator of the cable's energization status [18], [19] because acoustic measurement is nondestructive.

In an energized cable, whether or not it is carrying current, substantial second harmonic $(120-\mathrm{Hz})$ acoustic surface waves are generated. A piezoelectric accelerometer responds to both surface acoustic waves and power frequency electric fields of an energized conductor. Analysis of the frequency spectrum and spatial distribution of acoustic waves on the sensor surface is required to discriminate between the two effects [19]. Fig. 2 shows processed acoustic signatures of a three-phase 14-kV shielded distribution power cable in two states: nonenergized with strong ambient noise; and energized at low voltage, 10-A balanced load. The strong presence of the $120-\mathrm{Hz}$ component is fairly easy to detect; however, the presence of other energized cables in the vicinity of the cable under test makes the discrimination task more difficult. Surface imaging is necessary for nonambiguous classification.

\section{B. Evaluation of the Electrical Insulation Status}

Maintenance of aging power cables is a major cost item of the total maintenance of an electric network, which can be significantly reduced by a more accurate prediction of the remaining lifetime of cable insulation. Several methods are used to evaluate the aging status of electrical insulation, including eddy currents, acoustic sensing, and X-rays. The most useful and commonly used methods rely on measurement of electrical properties (dielectric conductivity and resistivity), measurement of partial discharge activity, and thermal analysis of insulation under stress.

1) Fringing Electric Field Dielectrometry: Interdigital dielectrometry is a subset of interdigital electrode sensor applications that relies on direct measurement of dielectric properties of insulating and semi-insulating materials from one side [20], [21]. The basic idea is to apply a spatially periodic electrical potential to the surface of the material under test. The combination of signals produced by the variation of the spatial period of interdigital electrodes, combined with the variation of electrical excitation frequency, potentially provides extensive information about the spatial profiles of the material under test. Since changes in the dielectric properties are usually induced by changes in various physical, chemical, or structural properties of materials, the dielectrometry measurements provide effective means for indirect nondestructive evaluation of vital parameters in industrial and scientific applications [22], [23].

While interdigital electrode structures have been used since the beginning of the century, the application of multiple penetration depth electric fields started in the 1960s [24]. Later, independent dielectrometry studies with single [25] and multiple [26] penetration depths using interdigital electrodes have been continued. Generally speaking, the evaluation of material properties with fringing electric fields is a much less developed area than comparable techniques. This field holds tremendous potential due to the inherent accuracy of capacitance and conductance measurements and to imaging capabilities combined with noninvasive measurement principles and model-based signal analysis.

A conceptual schematic of $\omega-k$ dielectrometry is presented in Fig. 3(a). For a homogeneous medium of semi-infinite extent, a periodic variation of electric potential along the surface in the $x$ direction produces an exponentially decaying pattern of electric fields penetrating into the medium in the $z$ direction. Fig. 3(b) shows the equivalent circuit of the sensor superimposed onto the schematic view of a half-wavelength cell. Note that each wavelength has an opposite conducting guard plane at the bottom of the substrate. For each wavelength, a follower op-amp drives the guard plane at the substrate bottom at the voltage $V_{G}=V_{S}$, thus eliminating any current between the sensing and guard electrodes.

The nondestructive measurement methods are often treated in the framework of the inverse problem theory. In our case, the definition of forward and inverse problems can be presented as shown in Fig. 4. For most applications, the inverse problem is 


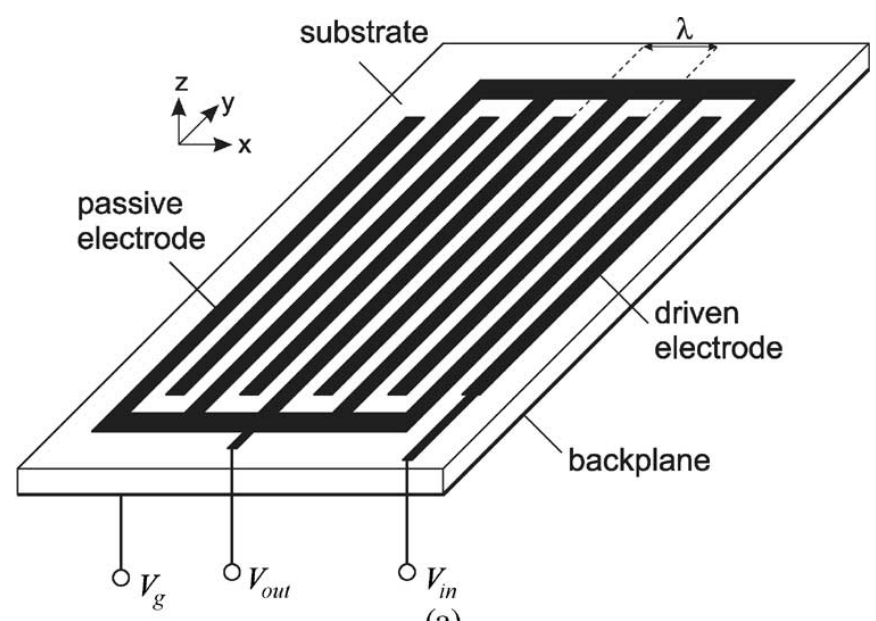

(a)

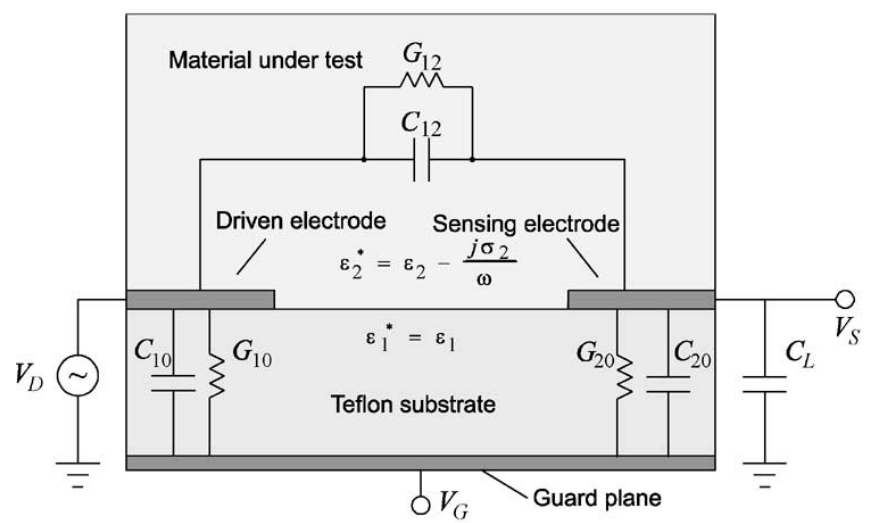

(b)

Fig. 3. Generic fringing electric-field interdigital sensor. The terminal characteristics, namely, capacitance and conductance between the interdigitated electrodes, are functions of the substrate and ambient environment properties. (a) A single-wavelength generic design. (b) Half-wavelength cross-section with a superimposed equivalent circuit model.

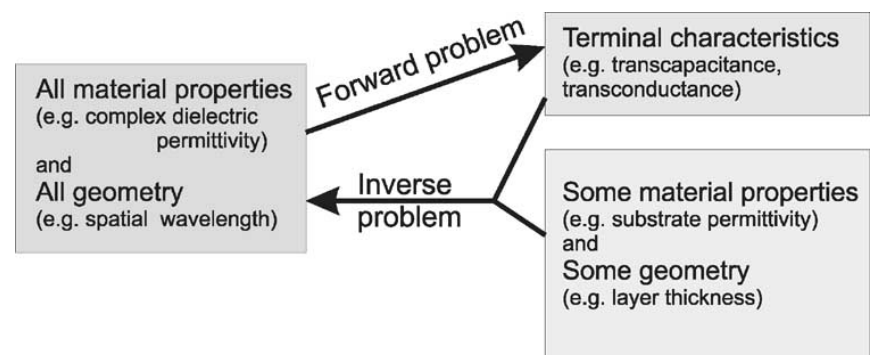

Fig. 4. Conceptual representation of forward and inverse problems in the framework of dielectrometry.

inherently more difficult. It does not necessarily have a unique or any solution, since it requires solving for unknown properties given a known subset of material and geometrical properties as well as the measured transcapacitance and transconductance. Furthermore, even if a unique and exact mathematical solution exists for a given set of input values, it may have no resemblance to the true physical parameters because of the effects of measurement noise.

2) Acoustic Sensing: Partial discharge (PD) measurement is an important diagnostic tool, especially for medium- and highvoltage cables, where local intensity of electric stress can reach breakdown values. A broad range of PD measurement techniques includes acoustic, current, and voltage [27], [28], electromagnetic [29], time- and frequency-domain reflectrometry [30], and optical sensing [31]. Acoustic sensing is very successful for switchgear and transformers [32], [33], because it is free from electrical interference, very easy to apply, has no need to power down, and does not require additional components, such as high-voltage capacitors [34]. However, cable applications with acoustic sensing are much more scarce [35], while electrical PD measurement methods are more preferred [36]. The main reason is that acoustical discharge signal is attenuated during propagation. Once the sensor can be delivered to a reasonable proximity of the discharge location (about $20 \mathrm{~m}$ ), both acoustic and RF pickup will become possible.

3) Thermal Analysis: Polymers commonly used as electrical insulation are thermally sensitive due to the limited strength of the covalent bonds that make up their structures. When exposed to sufficiently high temperatures that cause polymer degradation, these materials experience a drop in the glass transition temperature, which effectively lowers the upper service temperature and significantly reduces the room-temperature mechanical strength of these materials [37]. The lifetime of electrical insulation is reduced when a unit is subject to continual overheating, usually due to overload. The impregnated paper used in underground cables is particularly prone to aging through overheating, but it also holds true for all types of polymer insulation.

The insulation aging factors interact with each other. For example, thermal cycling may cause a loss of adhesion at an interface, thus creating a void within which PD will initiate, leading to electrical tracking and insulation failure [38]. So thermal analysis plays an important role in the evaluation of insulation status by supplying rich system information. Because of the no-contact measurement characteristics of the infrared (IR) sensor, it is one suitable sensor to detect the temperature distribution. By using it, the heat spot along the distribution system can be detected and analyzed.

\section{Water Uptake Measurement}

The presence of trees is usually accompanied by a high water uptake, on the order of $100 \mathrm{ppm}$ [39]. A highly polar material, water is best detectable by low-frequency dielectrometry techniques. Accurate measurement of moisture concentration across the thickness of oil-impregnated transformer pressboard is a significant challenge in the power industry. This task has been attacked for about two decades because the moisture diffusion processes are responsible for multimillion dollar losses due to explosions of large power transformers. The spatial moisture distribution has been measured successfully with a three-wavelength interdigital sensor [40].

Fig. 5 shows measured moisture profiles across the thickness of transformer pressboard for 14 distinct moments of time. The moisture spatial profiles were calculated from the measurement data, shown in Fig. 6, using multivariable parameter estimation algorithms for a three-wavelength sensor, combined with the moisture measurement data of the ambient environment (air relative humidity of $12 \%$ ), together with moisture equilibrium curves to give the pressboard moisture concentration of $1.8 \%$ at 


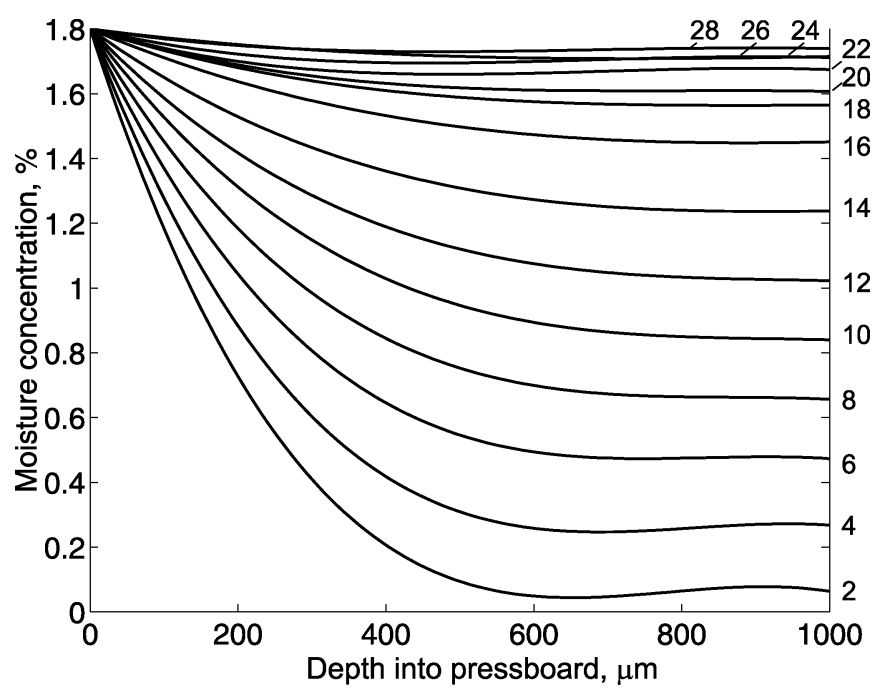

Fig. 5. Spatial profiles of moisture concentration across the thickness of pressboard. Numbers next to the curves correspond to the time in hours at which the profile was measured.

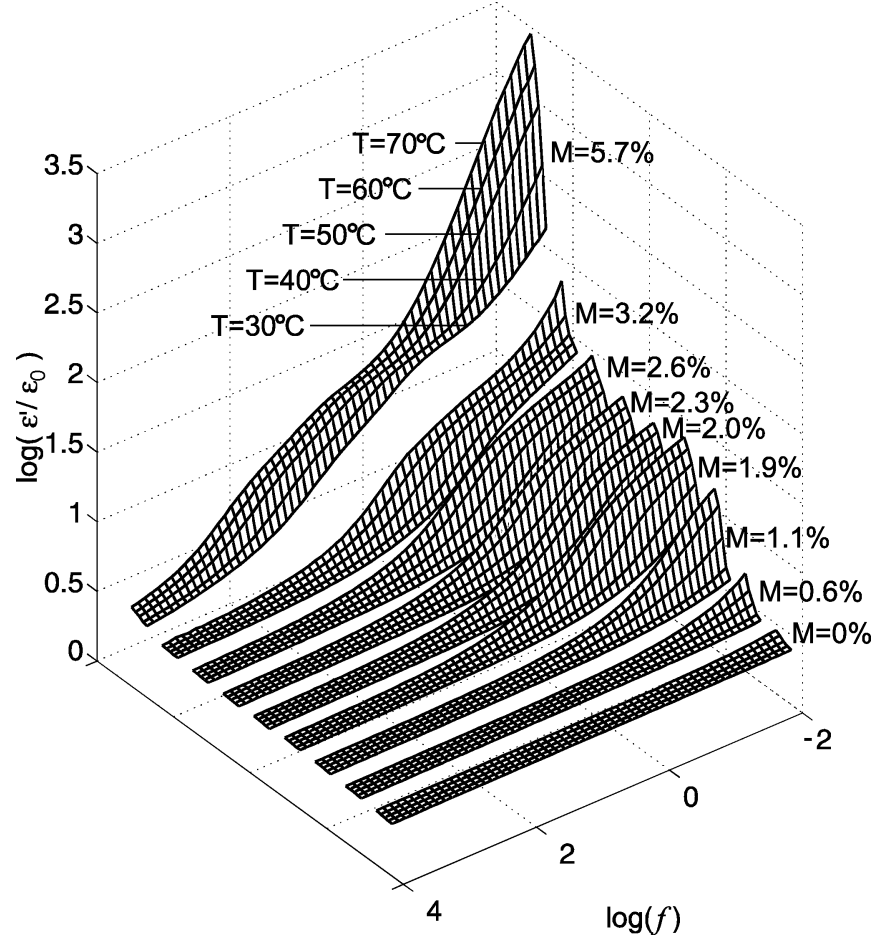

Fig. 6. Dielectric spectroscopy of oil-free Hi-Val pressboard for five temperatures and nine moisture levels at 61 frequencies.

$x=0$. The first two profiles (at the time moments of two hours and four hours) show a slightly overestimated amount of moisture in the middle region of the pressboard because the sensor resolution is insufficient to capture high profile gradients. The experiment is stopped at about $30 \mathrm{~h}$, when the signals indicate new steady state. At this point, the moisture profile is expected to be almost uniform and at the maximum value of $1.8 \%$.

\section{Parameter Estimation Algorithms}

One of the most significant challenges in multisensor systems is the processing of signals in real-time onboard. A deeper treatment of signal processing issues is outside of the scope of this paper. Included here is a commentary on computational challenges of multiprinciple sensor arrays. Through a generalized eigenvalue analysis and regularization theory, multivariable parameter estimation provides transformation of the vector of input parameters $\mathbf{X}$ into a vector of output signals $\mathbf{C}$, where the input parameters are physical variables of interest and the output signals are obtained from individual pixels of the sensor array. For linearized systems, this transformation can be expressed as

$$
A \cdot \mathbf{x}=\mathbf{C} .
$$

The coefficients of the square matrix $A$ are the partial derivatives of the individual output signals with respect to the input parameters

$$
a_{i j}=\frac{\partial C_{i}}{\partial x_{j}}
$$

Obtaining the physical variables of interest from the multidimensional sensor response means solving the inverse problem

$$
\mathbf{x}=A^{-1} \mathbf{C} \text {. }
$$

One of the most useful selectivity criteria is the condition number

$$
\nu(A)=\|A\| \cdot\left\|A^{-1}\right\|=\frac{\max \left|\lambda_{s}\right|}{\min \left|\lambda_{s}\right|}
$$

where $\lambda_{s}$ are eigenvalues of the $A$ matrix. The second equality holds only for eucledian norms. The physical meaning of (4) is the ratio of half axes of the ellipsoidal distribution of the vectors formed by the uncertainties of parameters of interest.

Eigenvalue theory can be used to optimize the $A$ matrix through modifications in sensor design and signal processing circuitry. Regularization methods trade off the accuracy of parameter estimation for numerical stability of the solution. Extensive additional efforts in this area are needed in regards to fringing electric field sensing. The potential of this approach will be unlocked once mathematically rigorous methods of signal analysis are implemented in software.

Mobility of sensor-carrying grippers makes possible perturbation position control, which provide additional sensitivity and selectivity improvement. The classic differential uncertainty analysis suggests that in a multiparameter measurement of dielectric permittivity $\varepsilon$, the measurement uncertainty is

$$
\frac{\Delta \varepsilon}{\varepsilon}=\frac{1}{\varepsilon} \sqrt{\sum_{i}\left[\left(\frac{d \varepsilon}{d x_{i}} \Delta x_{i}\right)^{2}\right]}
$$

where $x_{i}$ are individual sensor output variables (not parameters of interest, but intermediate variables). The dependence of $\varepsilon$ on $x_{i}$ is determined using numerical modeling or through analytical expressions.

The real measurement uncertainty due to geometry variations is very difficult to assess. By varying the sensor position by $\Delta x$, the device cannot only measure material properties, but also provide much more accurate estimation of measurement uncertainty. 


\section{CONCLUSION}

Review of monitoring technologies for maintenance of electric power system infrastructure suggests numerous advantages of mobile sensing. Miniaturization of mobile monitoring platforms is making realistic in-situ estimation of cable remaining life, operating conditions, and failure modes. A deeper understanding of physical nature of aging processes may be achieved through distributed sensing.

Several critical sensor technologies relevant to monitor the distribution system have been presented. They include acoustic sensing discrimination of energized cables, analysis of acoustic signatures of partial discharges, fringing electric field sensing, and infrared sensing. The main purpose of this multisensor system is to monitor the aging status, water uptake, and incipient faults in electrical insulation. The framework of multisensor signal processing challenges has been outlined as well.

\section{ACKNOWLEDGMENT}

The authors thank undergraduate students D. Bowman, R. Jose, H. Isern, and J. Chen for designing, assembling, and operating experimental setups. Research involvement of several undergraduate students has been supported by the Mary Gates Foundation. The authors also appreciate support of Southern California Edison, through work with A. Rodriguez, as well as technical assistance of the University of Washington Power Plant employees, especially M. Kirschenbaum.

\section{REFERENCES}

[1] J. Giddings, M. Simmons, and D. Hilder, "Practical experience on partial discharge measurement and location on power cables," in Proc. IEE Int. Conf. Partial Discharge, 1993, pp. 103-104.

[2] M. Tsutsui, H. Tsuchihashi, K. Satoh, M. Mukaida, H. Watanabe, S. Mori, Y. Kojima, and S. Yokoyama, "Manipulator system for constructing overhead distribution lines," IEEE Trans. Power Delivery, vol. 4, pp. 1904-1909, July 1989.

[3] S. Nio and Y. Maruyama, "Remote-operated robotic system for live-line maintenance work," in Proc. 6th Int. Conf. Transmission and Distribution Construction and Live Line Maintenance, 1993, pp. 425-435.

[4] M. Nakashima, H. Yakabe, Y. Maruyama, K. Yano, K. Morita, and H. Nakagaki, "Application of semi-automatic robot technology on hot-line maintenance work," in Proc. IEEE Int. Conf. Robotics and Automation, vol. 1, 1995, pp. 843-850.

[5] M. Boyer, "Systems integration in telerobotics: case study: maintenance of electric power lines," in Proc. IEEE Int. Conf. Robotics and Automation, vol. 2, 1996, pp. 1042-1047.

[6] A. Santamaria, R. Aracil, A. Tuduri, P. Martinez, F. Val, L. Penin, M. Ferre, E. Pinto, and A. Barrientos, "Teleoperated robots for live power lines maintenance (ROBTET)," in Proc. 14th Int. Conf. Exhibition on Electricity Distribution, vol. 3, 1997, pp. 31/1-31/5.

[7] J. Sawada, K. Kusumoto, Y. Maikawa, T. Munakata, and Y. Ishikawa, "A mobile robot for inspection of power transmission lines," IEEE Trans. Power Delivery, vol. 6, pp. 309-315, Jan. 1991

[8] H. Kobayashi, H. Nakamura, and T. Shimada, "An inspection robot for feeder cables-basic structure and control," in Proc. Int. Conf. Industrial Electronics, Control and Instrumentation, vol. 2, 1991, pp. 992-995.

[9] K. Suzumori, T. Miyagawa, M. Kimura, and Y. Hasegawa, "Micro inspection robot for 1-in pipes," IEEE/ASME Trans. Mechatron., vol. 4, pp. 286-292, Sept. 1999

[10] Y. Kawaguchi, I. Yoshida, H. Kurumatani, T. Kikuta, and Y. Yamada, "Internal pipe inspection robot," in Proc. IEEE Int. Conf. Robotics and Automation, 1995, pp. 857-862.

[11] M. Eghtesadi, "Inductive power transfer to an electric vehicle- analytical model," in Proc. III Int. Seminar/Workshop Direct and Inverse Problems of Electromagnetic and Acoustic Wave Theory, 1990, pp. 100-104.
[12] R. Severns, E. Yeow, G. Woody, J. Hall, and J. Hayes, “An ultra-compact transformer for a $100 \mathrm{~W}$ to $120 \mathrm{~kW}$ inductive coupler for electric vehicle battery charging," in Proc. 11th Annu. Applied Power Electronics Conf. Expo., vol. 1, 1996, pp. 32-38.

[13] J. A. Von Arx and K. Najafi, "On-chip coils with integrated cores for remote inductive powering of integrated microsystems," in Proc. Int. Conf. Solid State Sensors and Actuators, vol. 2, 1997, pp. 999-1002.

[14] A. Chandrakasan, R. Amirtharajah, S. Cho, J. Goodman, G. Konduri, J. Kulik, W. Rabiner, and A. Wang, "Design considerations for distributed microsensor systems," in Proc. Custom Integrated Circuits Conf., 1999, pp. $279-286$.

[15] M. Berger, O. Kubitz, and C. Pils, "Modeling mobile robot wireless real-time communication," in Proc. IEEE 47th Vehicular Technology Conf., vol. 3, 1997, pp. 2060-2064.

[16] T. Rupp, T. Cord, R. Lohnert, and D. Lazic, "Positioning and communication system for autonomous guided vehicles in indoor environment," in Proc. 9th Mediterranean Electrotechnical Conf., vol. 1, 1998, pp. 187-191.

[17] T. Walsh and T. Feldman, "Shielded cable is tested to determine if it is energized," Transm. Dist., pp. 48-51, July 1991.

[18] Z. Zabar, L. Birendbaum, B. R. Cheo, P. N. Joshi, and A. Spagnolo, "A detector to identify a de-energized feeder among a group of live ones," IEEE Trans. Power Delivery, vol. 7, pp. 1820-1824, Oct. 1992.

[19] A. V. Mamishev, S. X. Short, T. W. Kao, and B. D. Russell, "Non-intrusive sensing techniques for the discrimination of energized electric cables," IEEE Trans. Power Delivery, vol. 45, pp. 457-461, Apr. 1996.

[20] M. C. Zaretsky, P. Li, and J. R. Melcher, "Estimation of thickness, complex bulk permittivity and surface conductivity using interdigital dielectrometry," IEEE Trans. Elect. Insul., vol. 24, pp. 1159-1166, Dec. 1989.

[21] J. O. Simpson and S. A. Bidstrup, "Modeling conductivity and viscosity changes during epoxy cure using TEA, DMA, and DSC," in Proc. Amer. Chem. Soc., Div. Polymeric Materials: Science and Engineering, Fall Meeting, vol. 69, 1993, pp. 451-452.

[22] N. J. Goldfine, A. P. Washabaugh, J. V. Dearlove, and P. A. von Guggenberg, "Imposed $\omega-k$ magnetometer and dielectrometer applications," in Review of Progress in Quantitative Nondestructive Evaluation, D. Thompson and D. Chimenti, Eds. New York: Plenum, 1993, vol. 12.

[23] Y. K. Sheiretov and M. Zahn, "Dielectrometry measurements of moisture dynamics in oil-impregnated pressboard," IEEE Trans. Dielect. Elect. Insul., vol. 2, pp. 329-351, June 1995.

[24] I. G. Matis, "On multiparameter control of dielectric properties of laminate polymer materials," Latvijas PSR Zinatnu Akademijas Vestis, Fizikas un Tehnisko Zinatnu Serija, no. 6, pp. 60-67, 1966.

[25] S. D. Senturia and C. M. Sechen, "The use of the charge-flow transistor to distinguish surface and bulk components of thin-film sheet resistance," IEEE Trans. Electron Devices, vol. ED-24, p. 1207, Sept. 1977.

[26] R. S. Jachowicz and S. D. Senturia, "A thin-film capacitance humidity sensor," Sens. Actuators, vol. 2, pp. 171-186, Dec. 1981.

[27] B. T. Phung, T. R. Blackburn, and R. E. James, "On-line partial discharge measurement on high voltage power cables," in Proc. 11th Int. Symp. High Voltage Engineering, 1999, pp. 328-332.

[28] J. P. Steiner, P. H. Reynolds, and W. L. Weeks, "Estimating the location of partial discharges in cables," IEEE Trans. Elect. Insul., vol. 27, pp. 44-59, Feb. 1992.

[29] U. Schichler, "A sensitive method for on-site partial discharge detection on XLPE cable joints," in Proc. 5th Int. Conf. Properties and Applications of Dielectric Materials, 1997, pp. 1099-1102.

[30] E. Gulski, F. Wester, J. Smit, P. Seitz, and M. Turner, "Advanced partial discharge diagnostic of MV power cable system using oscillating wave test system," IEEE Electr. Insul. Mag., vol. 16, pp. 17-25, Mar. 2000.

[31] R. J. Van Brunt, K. L. Stricklett, J. P. Steiner, and S. V. Kulkarni, "Recent advances in partial discharge measurement capabilities at NIST," IEEE Trans. Elect. Insul., vol. 27, pp. 114-129, Feb. 1992.

[32] Y. Lu, X. Tan, and X. Hu, "PD detection and localization by acoustic measurements in an oil-filled transformer advanced partial discharge diagnostic of MV power cable system using oscillating wave test system," in Proc. Inst. Elect. Eng., Sci., Measurement Technol., vol. 147, Mar. 2000, pp. 81-85.

[33] IEEE Trial-Use Guide for the Detection of Acoustic Emissions for Partial Discharges in Oil-Immersed Power Transformers, IEEE Std. C57.127-2000, 2000.

[34] Y. Tian, P. Levin, A. Davies, and G. Hathaway, "Acoustic emission techniques for partial discharge detection within cable insulation," in Proc. Inst. Elect. Eng. Eighth Int. Conf. Dielectric Materials, Measurements and Applications, 2000, pp. 503-508.

[35] R. Heinrich, R. Jobava, W. Kalkner, and A. Gheonjian, "Investigation and optimization of a sensor for partial discharge detection on high voltage XLPE cables," in Proc. III Int. Seminar/Workshop Direct and Inverse Problems of Electromagnetic and Acoustic Wave Theory, 1998, pp. 134-137. 
[36] B. Oyegoke, P. Hyvonen, and M. Aro, "Partial Discharge Measurement as Diagnostic Tool for Power Cable Systems," Helsinki Univ. Technol., Tech. Rep., Espoo, Finland, 2001.

[37] N. Grassie and G. Scott, Polymer Deregulation and Stabilization. Cambridge, U.K.: Cambridge Univ. Press, 1929.

[38] J. Densley, "Ageing mechanisms and diagnostics for power cables-an overview," IEEE Elect. Insul. Mag., vol. 17, no. 1, pp. 1820-1824, 2001.

[39] R. Clavreul, J. M. Fourmigue, F. Duchateau, J. Berdala, and J. L. Parpal, "Estimating the location of partial discharges in cables," in Proc. Conf. Electrical Insulation and Dielectric Phenomena, 1992, pp. 650-655.

[40] A. V. Mamishev, Y. Du, B. C. Lesieutre, and M. Zahn, "Development and applications of fringing electric field sensors and parameter estimation algorithms," J. Electrostatics, vol. 46, no. 2, pp. 109-123, 1999.

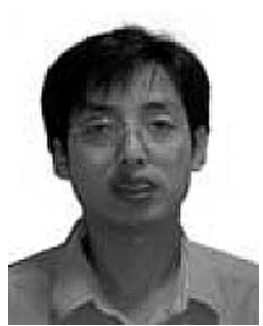

Bing Jiang (S'01) received the B.S. degree from Tianjin University, Tianjin, China, in 1995 and the M.S. degree in electrical engineering from the University of Washington, Seattle, in 2003.

Currently, he is a Graduate Student in the Department of Electrical Engineering, University of Washington, Seattle. He is an author of several journal and conference papers. His research interests include robotics, power, and sensors.

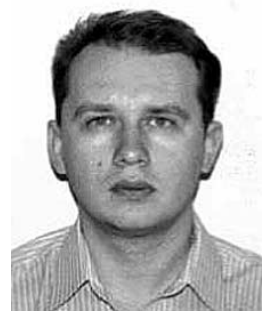

Alexander Mamishev (S'92-M'00) received the B.S. degree in electrical engineering from the Kiev Polytechnic Institute, Kiev, Ukraine, in 1992, the M.S. degree in electrical engineering from Texas A\&M University, College Station, in 1994, and the $\mathrm{Ph} . \mathrm{D}$. degree in electrical engineering from the Massachusetts Institute of Technology, Cambridge, in 1999.

Currently he is an Assistant Professor and Director of Sensors, Energy, and Automation Laboratory (SEAL) in the Department of Electrical Engineering at the University of Washington, Seattle. He is the author of several technical publications. His research interests include sensor design and integration, dielectrometry, electrical insulation diagnostics, and power quality.

Dr. Mamishev is a recent recipient of the National Science Foundation CAREER Award, Outstanding IEEE Student Branch Counselor Award, and Outstanding Research Advisor Award. He is a reviewer for the IEEE TRANSACTIONS ON POWER DELIVERY and an Associate Editor for the IEEE TRANSACTIONS ON DIELECTRICS AND ELECTRICAL INSULATION. 